\title{
The unique functions of tissue-specific proteasomes
}

\author{
Andrea Kniepert ${ }^{1}$ and Marcus Groettrup ${ }^{1,2}$ \\ ${ }^{1}$ Division of Immunology, Department of Biology, University of Konstanz, D-78457 Konstanz, Germany \\ ${ }^{2}$ Biotechnology Institute Thurgau at the University of Konstanz, CH-8280 Kreuzlingen, Switzerland
}

\begin{abstract}
The $26 \mathrm{~S}$ proteasome is the main protease in eukaryotes. Proteolysis occurs within the cylindrical 20S proteasome that is constitutively expressed in most tissues. However, three tissue-specific versions of the $20 \mathrm{~S}$ proteasome have been discovered to date. The immunoproteasome is optimized to process antigens and it directs the differentiation of T helper (Th) cells. The thymoproteasome is selectively expressed in cortical epithelial cells of the thymus where it plays an essential role in the positive selection of T lymphocytes. Finally, the spermatoproteasome is found in the testes where it is required during spermatogenesis. Here, we outline how tissue-specific proteasomes adapt to functional needs in their respective tissues and how their selective inhibition may be used to interfere with autoimmune diseases and cancer.
\end{abstract}

\section{The proteasome: evolution towards complexity and specialization}

The proteasome is an ancient enzyme that has steadily evolved towards a higher complexity of subunits and regulators while preserving its cylindrical architecture constituted from four stacked rings. In the archebacterium Thermoplasma acidophilum, the $20 \mathrm{~S}$ proteasome consists of two outer rings with seven copies of a single $\alpha$-type subunit and two inner rings with seven copies of the same $\beta$-type subunit [1] (Figure 1). In the eubacterial actinomycete Rhodococcus sp., the $20 \mathrm{~S}$ proteasome is constructed from two different $\alpha$-type and two different $\beta$-type subunits [2], whereas in the eukaryotic yeast Saccharomyces cerevisiae, it consists of seven different $\alpha$-type and seven different $\beta$-type subunits [3]. Proteolysis takes place in the inner chamber of the proteasome formed by the two $\beta$ rings. Although each of the $\beta$ subunits of the archebacterial and eubacterial proteasomes carry peptidolytically active centers, only three of the seven eukaryotic $\beta$-type subunits are catalytically active. It appears that the increase in complexity is accompanied with a reduction in the number of active $\beta$ subunits; the driving force for which has remained elusive. This tendency goes along with a specialization of substrate specificity of these three $\beta$ subunits, which is measured via the hydrolysis of small fluorogenic peptides with certain amino acids in the $\mathrm{P} 1$ position. These define the chymotrypsin-like activity (cleavage after hydrophobic residues), the trypsin-like activity (cleavage after basic residues), and the caspase-like activity of the proteasome (cleavage after acidic residues). Although the archaeal and bacterial proteasomes only show chymotrypsin-like activity, the eukaryotic proteasomes show all three activities that are associated with the $\beta 1$ (caspase-like activity), $\beta 2$ (trypsin-like activity), and $\beta 5$ (chymotrypsin-like activity) subunits. These $\beta$-type subunits of eukaryotes have been named according to their position in the $\beta$ ring [3] (Figure 1).

Interestingly, for the normal housekeeping function of the proteasome in proteolysis of regulatory or misfolded proteins, this diversification is not necessary. Yeast mutants that only harbor the chymotrypsin-like activity remained viable [4]. Whether specialized proteasome subunits are needed to cope with an enhanced protein degradation load is currently debated (see below). However, when looking at the peptide products of the proteasome, the cleavage preferences of $\beta 1, \beta 2$, and $\beta 5$ make a big difference. Proteasome products are used by the immune system to enable antigen recognition by $\mathrm{T}$ lymphocytes and proteasomal peptide products may even have as yet unknown signaling functions in spermatogenesis or other aspects of cell biology. The functional specialization of $\beta 1, \beta 2$, and $\beta 5$ enables the modification of the cleavage preferences of the proteasome by replacing them with inducible or tissue-specific homologs. Next to the $\beta 1-\beta 2-$ $\beta 5$ containing 'constitutive' proteasome, three further proteasome subtypes with differing subunit composition have been found: the immunoproteasome, the thymoproteasome, and the spermatoproteasome (Figure 2). Recent insights, such as how their special subunit composition enables them to serve functions that the constitutive proteasome can only exert suboptimally, are discussed in this review.

The immunoproteasome in antigen processing

The immunoproteasome is a tissue-specific complex because it is continuously expressed in cells of the immune system such as T cells, B cells, monocytes, macrophages, dendritic cells, or medullary thymic epithelial cells [5]. However, it is also strongly inducible by the proinflammatory cytokines interferon (IFN)- $\gamma$ and tumor necrosis factor (TNF)- $\alpha$ in virtually all tissues except for the brain, where 




Archaebacterial proteasome



Bacterial proteasome (actinomycetales)

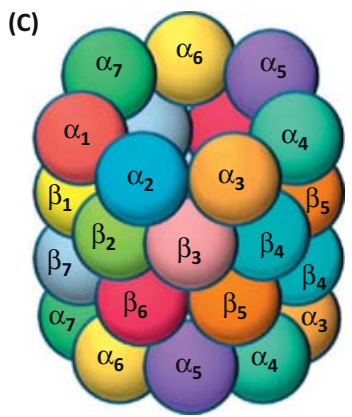

Eukaryotic proteasome

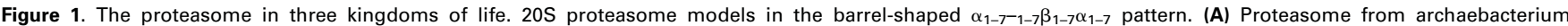
Thermoplasma acidophilum. The archaebacterial proteasome is composed of two outer and two inner rings; each of which contains seven identical $\alpha$ subunits (in red) or $\beta$ subunit (gray), respectively. (B) Among bacteria, proteasomes have been found in eubacterial actinomycetales. The proteasome from Rhodococcus erythropolis is built of two different $\alpha$ (red and orange) and two different $\beta$ subunits (gray and green), which are most likely randomly arranged in the respective $\alpha$ and $\beta$ rings. (C) Proteasome from the eukaryote Saccharomyces cerevisiae. Seven different $\alpha$ and seven different $\beta$ subunits have their defined positions in the eukaryotic $20 \mathrm{~S}$ proteasome.

immunoproteasome expression is largely confined to microglia and invading leukocytes [6]. Remarkably, immunoproteasomes replace up to $90 \%$ of constitutive proteasomes in tissues in the course of viral, bacterial, or fungal infections [5-7], which raises the question why the immune system induces such an extensive change in proteasome composition. Interestingly, this argues that immunoproteasomes can take over the housekeeping functions of constitutive proteasomes for several days without harming the inflamed tissues.

In the immunoproteasome all three active site bearing subunits of the constitutive 20 S proteasome ( $\beta 1, \beta 2$, and $\beta 5$ ) are replaced by homologous, cytokine-inducible subunits named $\beta 1 \mathrm{i}$ [low molecular mass polypeptide (LMP)2], $\beta 2 \mathrm{i}$ [(multicatalytic endopeptidase complex-like-1 (MECL-1)], and $\beta 5 \mathrm{i}$ (LMP7), respectively. Two subunits of the immunoproteasome, $\beta 1 \mathrm{i}$ and $\beta 5 \mathrm{i}$, were discovered during genomic sequencing of the major histocompatibility complex (MHC) gene locus over two decades ago, whereas the $\beta 2 \mathrm{i}$ subunit was discovered later because it is not encoded in the MHC locus [8]. Many members of the MHC class I and II antigen presentation processing pathways are encoded within the MHClocus, which had focused immunoproteasome research on their role in antigen processing [9].

Proteasome activity is required for MHC class I but not class II restricted antigen presentation. This was shown with broad spectrum proteasome inhibitors that interfered with MHC class I release from the endoplasmic reticulum (ER) and class I cell surface expression [10-12], whereas the classical MHC class II pathway was barely affected by them. The proteasome generates peptide fragments from protein antigens that bind into the peptide-binding groove of class I molecules. They can be generated in their final length by proteasomes either in the cytoplasm or in the nucleus. They can also be generated as N-terminally extended precursors that are subsequently trimmed either in the cytoplasm or after transport into the lumen of the ER where they meet partially folded MHC class I/ $\beta 2$-microglobulin complexes [8]. Only if a given peptide binds tightly into the peptide ligand cleft of class I molecules does the trimeric complex finally fold and get released from the ER to the cell surface. There, the antigen receptor of cytotoxic T lymphocytes can recognize peptide plus MHC and kill the cell that presents their cognate antigens. The criteria for tight peptide-class I binding are (i) a length of 8-9 amino acids and (ii) the presence of anchor residues at the $\mathrm{C}$ terminus of the peptide and somewhere else within the peptide sequence. The C-terminal anchor residues in humans can be of basic or hydrophobic nature, whereas mouse class I molecules only accept peptides with hydrophobic $\mathrm{C}$ termini. These rather tight acceptance criteria render it difficult to cleave out a sufficient number of peptide ligands to mount an immune response especially against small viruses that encode only a few proteins. It is certainly one of the contributions of the immunoproteasome to a successful cellular immune response against viruses or intracellular bacteria that its altered cleavage priorities enhance the versatility of antigen processing and the chance to generate a pivotal antigenic peptide [8].

The recently reported high-resolution X-ray crystallographic structures of the mouse constitutive proteasome and immunoproteasome allow a side by side comparison of the three different substrate-binding pockets that define the cleavage priorities of the two complexes [13]. The most striking difference is that between $\beta 1$ and $\beta 1$. Although the former accommodates peptides with an acidic $\mathrm{P} 1$ residue (caspase-like activity), the latter binds peptides with a hydrophobic $\mathrm{P} 1$ residue. For unknown reasons, the hundreds of allelic variants of mouse and human MHC class I molecules do not accept peptide ligands with acidic $\mathrm{C}$-terminal anchor residues. The proteasome is the main determiner of the $\mathrm{C}$ terminus of antigenic peptides, therefore, $\beta 1$ products are lost for antigen presentation. The replacement of $\beta 1$ with $\beta 1 \mathrm{i}$ avoids this loss, generating useful class I ligands with hydrophobic $\mathrm{C}$ termini, therefore enough ligands become available to sustain the approximately tenfold upregulation of class I cell surface expression after stimulation with IFN- $\gamma$. This advantage most likely explains, at least in part, a phenotype of immunoproteasome-deficient mice: they show a $50 \%$ reduced MHC class I surface expression of lymphocytes and dendritic cells [14-16]. Also the replacement of $\beta 5$ by $\beta 5 \mathrm{i}$ 
(A)

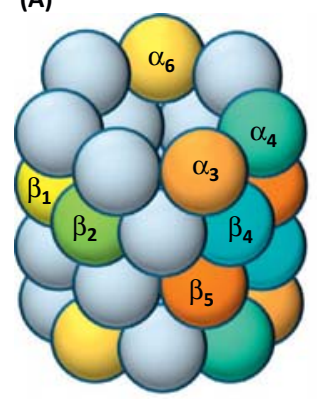

(B)

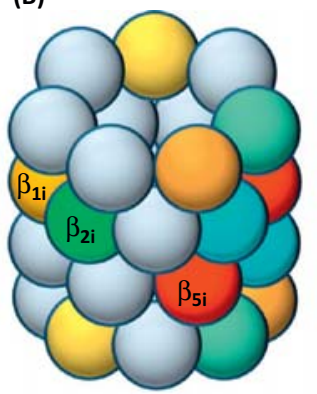

(C)

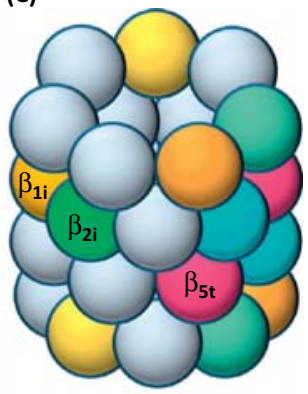

(D)

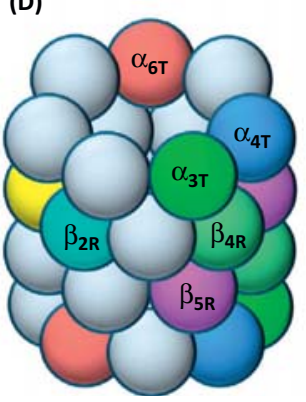

(E)

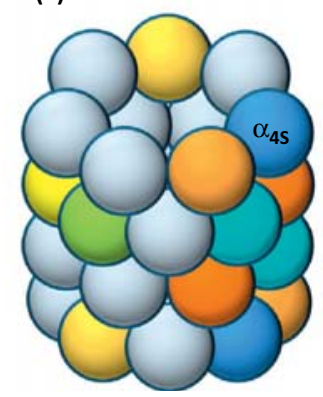

Figure 2. Eukaryotic $20 \mathrm{~S}$ proteasome subtypes. (A) Constitutive proteasome; subunits that differ in tissue specific 20S proteasomes are highlighted in different colors. (B) Vertebrates possessing an adaptive immune system can additionally express the inducible proteolytic subunits $\beta 1 \mathrm{i}$ [low molecular mass polypeptide (LMP) 2], $\beta 2 \mathrm{i}$ (multicatalytic endopeptidase complex-like-1; MECL-1), and $\beta 5 \mathrm{i}$ (LMP7) that are incorporated into immunoproteasomes. (C) The thymoproteasome expressed in cortical thymic epithelial cells (cTECs) contains the proteolytic subunits $\beta 1 \mathrm{i}, \beta 2 \mathrm{i}$, and the thymus-specific subunit $\beta 5$ t. (D) Spermatoproteasomes from Drosophila melanogaster are composed of several alternative subunits. Testis-specific subunits are: $\alpha 3 T, \alpha 4 T 1 / 2, \alpha 6 \mathrm{~T}, \beta 2 \mathrm{R} 1 / 2, \beta 4 \mathrm{R} 1 / 2$, and $\beta 5 \mathrm{R} 1 / 2$. (E) Mammalian spermatoproteasomes characteristically incorporate the testis-specific $\alpha 4$ s subunit.

likely contributes to this phenomenon because the $\mathrm{S} 1$ pocket of $\beta 5 \mathrm{i}$ better accommodates larger hydrophobic amino acid side chains, which are the preferred C-terminal anchor residues for many class I molecules. Moreover, $\beta 5 \mathrm{i}$ has a more hydrophilic surrounding for the active site threonine and forms a specific hydrogen bond that stabilizes the tetrahedral transition state during catalysis, which could together kinetically favour the peptidolytic activity of $\beta 5 i$ [13]. By contrast, the replacement of $\beta 2$ with $\beta 2 \mathrm{i}$ is enigmatic, because both substrate pockets are virtually identical and they show the same trypsin-like activity $[17,18]$. Nevertheless, $\beta 2 i$ knockout mice have clear phenotypes (see below), which may have a structural rather than a catalytic basis $[19,20]$.

Compared to the relatively minor consequence of immunoproteasome deficiency on bulk MHC class I peptide loading and surface expression, the loss of $\beta 1 i, \beta 2 i$, or $\beta 5 i$ can have enormous positive and negative consequences for single epitopes. Some epitopes are immunoproteasome dependent in their generation [21-24], whereas others - including important $\mathrm{T}$ cell epitopes of tumor antigens - are destroyed by immunoproteasomes [25,26]. A special case, where the expression of a catalytically inactive $\beta 1 \mathrm{i}$ subunit could rescue an immunoproteasome-dependent $\mathrm{T}$ cell epitope, was recently explained: the inactive $\beta 1 \mathrm{i}$ takes the place of $\beta 1$, which would have destroyed the epitope with its caspaselike activity [27]. Previously, the impact of immunoproteasomes on the peptide repertoire presented by MHC class I molecules has generally been underestimated [28]. The proteomic analysis of peptides eluted from class I molecules of splenocytes from mice either proficient or deficient for all three immunoproteasome subunits revealed that about one-third of peptides were only presented by wild type cells, one-third were presented only by $\beta 1 \mathrm{i}^{-/-} \beta 2 \mathrm{i}^{-/-} \beta 5 \mathrm{i}^{-/-}$cells, and another third were presented by splenocytes from both mouse strains [16]. This result implies that more $\mathrm{T}$ cell epitopes must be affected by immunoproteasome induction in inflamed tissues than previously expected.

Initial reports of the biological effects of immunoproteasome deficiency were disappointing. Lymphocytic choriomeningitis virus was cleared normally in immunoproteasomedeficient mice [15], whereas there was a defect reported to clear Listeria monocytogenes from the liver but not the spleen of $\beta 5 \mathrm{i}^{-/-}$mice [29]. However, a recent study revealed that $\beta 5$ i-deficient mice succumb to an infection with Toxoplasma gondii that wild type mice can survive [30]. Therefore, it is warranted to test further pathogens in immunoproteasome-deficient mice including the recently generated triple knockout mice. If more pathogens that rely on immunoproteasomes for their clearance can be identified, and if immunoproteasomes fulfill the proteolytic housekeeping functions as efficiently as constitutive proteasomes, one may wonder why immunoproteasomes are not constitutively expressed in all tissues.

Several years ago we proposed the hypothesis that the induction of immunoproteasome expression in inflamed tissues would focus the effector phase of the cytotoxic $\mathrm{T}$ cell response on immunoproteasome-dependent epitopes that would not be presented in uninflamed tissues. This could prevent accidentally activated self-reactive bystander 
$\mathrm{T}$ cells in inflamed tissues from finding the same selfepitopes elsewhere in the body [31]. We predicted that immunoproteasomes would thus contribute to protection from autoimmune diseases. Recently, Zaiss et al. reported evidence supporting this hypothesis. They showed that $\beta 2 \mathrm{i}^{-/-} \beta 5 \mathrm{i}^{-/-}$mice, when irradiated and reconstituted with bone marrow from the same strain, developed insulindependent diabetes mellitus that was caused by cytotoxic Tlymphocytes recognizing $\beta$-islet antigens [32]. Moreover, it was shown that certain allelic variants of $\beta 5 \mathrm{i}$ or $\beta 1 \mathrm{i}$ were associated with human type 1 diabetes, and other human autoimmune or autoinflammatory diseases [33-36]. Taken together, it appears that on the one hand the immunoproteasome enhances and diversifies the set of class I ligands and on the other hand it may contribute to the avoidance of autoimmunity.

\section{The immunoproteasome in Th cell differentiation and the pathogenesis of autoimmunity}

A new unexpected function of the immunoproteasome, which is unrelated to its role in antigen processing, has recently been discovered. When $\mathrm{T}$ cells lacking $\beta 2 \mathrm{i}, \beta 5 \mathrm{i}$ or, to a lesser extent, $\beta 1 \mathrm{i}$ were transferred into virus-infected recipient mice, they failed to survive in contrast to immunoproteasome-proficient T cells $[19,23]$. This was true for both Th cells and cytotoxic T cells, therefore, this phenomenon could not be related to MHC class I antigen processing. The notion that $\mathrm{T}$ cells need the immunoproteasome to survive in an inflammatory environment sparked the idea that a selective inhibition of $\beta 5 \mathrm{i}$ may interrupt the pathogenetic function of $\mathrm{T}$ cells in chronic inflammatory diseases like autoimmune diseases. Remarkably, the treatment of mice with the $\beta 5 \mathrm{i}$ selective inhibitor ONX 0914 (formerly called PR-957) was able to prevent the progression of rheumatoid arthritis in two preclinical mouse models and the induction of $\mathrm{T}$ cell-mediated type 1 diabetes [37]. Moreover, the inhibition or the deletion of $\beta 5 \mathrm{i}$ or $\beta 2 \mathrm{i}$ abrogated the dextran-sulfate-sodium-mediated induction of colitis $[20,38,39]$, and $\beta 5$ i inhibition prevented the development of lupus-erythematosus-like disease in lupusprone mice [40]. These findings may open a new approach for the treatment of proinflammatory autoimmune diseases using immunoproteasome selective inhibitors. In addition, they raise the interesting question whether the immunoproteasome is mechanistically involved in these diseases. A first step in support of this idea may be the observation that the inhibition or deletion of $\beta 5 \mathrm{i}$ partially suppressed the differentiation of the proinflammatory Th1- and Th17-type cells, while the development of antiinflammatory regulatory $\mathrm{T}$ (Treg) cells was suppressed [41]. Both Th1 and Th17 cells are involved in the development of autoimmune diseases, whereas Treg cells suppress them. Mechanistically, silencing $\beta 5$ i suppresses phosphorylation of the transcription factors signal transducer and activator of transcription (STAT) 1 and STAT3, which drive the differentiation of naïve T helper cells to Th1 and Th17 cells, respectively $[41,42]$. How exactly $\beta 5$ i enables STAT1 and STAT3 phosphorylation remains to be determined. An intriguing hypothesis is that $\beta 5 \mathrm{i}$ processes a factor that promotes Th1 and Th17 differentiation differently than $\beta 5$. In general, the proteasome is able to generate factors from precursor proteins by limited proteolysis as has been shown for the nuclear factor $(\mathrm{NF})-\kappa \mathrm{B}$ precursor protein p105 [43] and the plasma membrane resident transcription factor precursor Spt23/MGA2 [44]. In fact, it has been reported that processing of p105 to p50 is specifically performed by the immunoproteasome [45], but this concept could not be confirmed by others $[46,47]$ and remains a matter of experimentation and debate.

\section{A special role of the immunoproteasome in the accelerated degradation of polyubiquitin conjugates?}

Still another special function of the immunoproteasome is the enhanced degradation of polyubiquitin conjugates, which was recently proposed by Seifert et al. [48]. It was reported that after stimulation of fibroblasts or HeLa cells with IFN- $\gamma$ polyubiquitin conjugates accumulated for $8 \mathrm{~h}$ and declined thereafter. The decline of ubiquitin conjugates correlated with the induction of immunoproteasomes in these cells, which led to the hypothesis that immunoproteasomes might be needed to handle the excess of ubiquitin conjugates in IFN- $\gamma$-stimulated cells. Such a transient rise and decline in polyubiquitin conjugates was not observed by Seifert et al. in IFN- $\gamma$-stimulated mouse embryonal fibroblasts (MEFs) from $\beta 5$ i-deficient mice, supporting this concept. Instead, an accumulation of ubiquitin-containing protein aggregates was observed in $\beta 5 \mathrm{i}$-deficient MEFs as well as in the brain of $\beta 5 \mathrm{i}^{-1-}$ mice suffering from experimental autoimmune encephalomyelitis (EAE); a model for multiple sclerosis. In apparent agreement with the latter result, higher EAE disease scores were recorded for $\beta 5 \mathrm{i}^{-1-}$ as compared to wild type mice. The notion that the $26 \mathrm{~S}$ immunoproteasome would degrade polyubiquitylated proteins faster than the constitutive $26 \mathrm{~S}$ proteasome was then confirmed by monitoring the degradation of polyubiquitylated mucin by the two proteases in vitro [48].

That IFN- $\gamma$ stimulation might induce a protease that would more efficiently degrade the misfolded proteins that it also induces is an appealing notion. However, none of the aforementioned results by Seifert et al. [48] that indicated a higher capacity of the immunoproteasome to degrade polyubiquitylated proteins could be confirmed in the same or very similar experiments performed by Nathan et al. [49]. Instead, it was found that the immunoproteasome and the constitutive proteasome do not differ in their ability to bind and to degrade polyubiquitylated proteins. To identify details in experimental procedures that may account for the strikingly different outcomes of the experiments in these two studies is beyond the scope of this review. However, it should be mentioned that no difference in the amounts of polyubiquitylated proteins in splenocytes from wild type mice and mice triply deficient for $\beta 1 \mathrm{i}$, $\beta 2 \mathrm{i}$, and $\beta 5 \mathrm{i}$ could be detected by Rock and colleagues [16]. Furthermore, there is no evidence that immunoproteasomes associate preferentially with any of the known proteasome regulators including the $19 \mathrm{~S}$ regulator, which polyubiquitylated proteins need to dock onto in order to be unfolded under ATP hydrolysis to enter the $20 \mathrm{~S}$ proteasome or 20S immunoproteasome [50]. This is consistent with the recently elucidated high resolution crystal structures of mouse constitutive- and immunoproteasomes, 
which structurally do not differ in their $\alpha$-endplates where all known regulators bind [13]. Therefore, it is difficult to envision how immunoproteasomes would degrade polyubiquitylated proteins faster than constitutive proteasomes do. Taken together, structural considerations and compelling experimental data $[16,49]$ argue that immunoproteasomes do not degrade ubiquitin conjugates faster than constitutive proteasomes.

\section{The role of the thymoproteasome in selecting developing $\mathrm{T}$ Iymphocytes}

Sometimes data mining can be more rewarding than gold mining. When Shigeo Murata, Keiji Tanaka, and colleagues were searching a genome data base for proteasomerelated genes they found an open reading frame with homology to $\beta 5$ and $\beta 5 \mathrm{i}$. This gene was exclusively expressed in the thymus, which led to its designation $\beta 5 \mathrm{t}$ ( $\mathrm{t}$ for thymus) [51]. A more detailed analysis of subpopulations of cells in the thymus revealed that $\beta 5 \mathrm{t}$ was exclusively expressed in epithelial cells of the thymic cortex. The thymus is a primary lymphoid organ where immature $\mathrm{T}$ cells gain their antigen receptor specificity and where those $\mathrm{T}$ cells that bind to the MHC molecules encoded by the individual (self-MHC) are positively selected to survive and mature [52]. This positive selection occurs in the cortex of the thymus and is mediated by the highly specialized cortical thymic epithelial cells (cTECs). By contrast, $\mathrm{T}$ cells that recognize self-MHC and a self-peptide have the potential to cause autoimmunity, thus they need to be selected against and induced to die by apoptosis. This process of negative selection is mediated by medullary thymic epithelial cells (mTECs) and dendritic cells, which both express about equivalent levels of constitutive and immunoproteasomes [53,54]. To determine the function of the new proteasome subunit $\beta 5 \mathrm{t}$, Murata et al. generated a $\beta 5$ t-deficient mouse that turned out to have a dramatic phenotype. Although the selection of mature $\mathrm{CD} 4^{+} \mathrm{T}$ helper cells was normal, the selection of mature $\mathrm{CD}^{+}$cytotoxic T cells was reduced by $80 \%$, leading to a similarly strong reduction of $\mathrm{CD}^{+} \mathrm{T}$ cells in the spleen [51]. Consequently, $\beta 5 \mathrm{t}^{-1-}$ mice succumbed to an influenza virus infection that wild type mice survived [55].

The strong phenotype of $\beta 5 \mathrm{t}^{-/-}$mice raised the question of why the $\beta 5$ or $\beta 5$ i subunits cannot compensate for the loss of $\beta 5 \mathrm{t}$. A problem with proteasome assembly could be excluded because with $\beta 5 \mathrm{t}$ deficiency the so-called thymoproteasome of mouse cTECs uses the $\beta 5 i$ active site subunit in its place [55]. Insight into the unique function of $\beta 5 \mathrm{t}$ in thymic positive selection was derived when comparing the peptidolytic activities of $\beta 5 \mathrm{t}$ - and $\beta 5$-containing proteasomes. The $\beta 5 \mathrm{t}^{+}$proteasomes had a much lower chymotrypsin-like activity than $\beta 5^{+}$proteasomes, whereas their caspase-like and trypsin-like activities were not affected by $\beta 5 t$ incorporation into the proteasome [51]. This change in activity profile can be explained when looking at the amino acids that line the substrate binding pocket of $\beta 5 \mathrm{t}$. The S1 pockets of $\beta 5$ and $\beta 5 \mathrm{i}$ accommodate larger hydrophobic amino acids, whereas four replacements of hydrophobic amino acids in the S1 pocket of $\beta 5$ and $\beta 5 \mathrm{i}$ by the hydrophilic amino acids serine or threonine in $\beta 5 \mathrm{t}$ predict a cleavage preference for $\beta 5 \mathrm{t}$ after hydrophilic or less hydrophobic amino acids. The resulting peptide products of $\beta 5 \mathrm{t}^{+}$proteasomes are expected to be weak ligands of mouse MHC class I molecules. Murata et al. proposed that such weak $\beta 5$ t-dependent class I ligands, which have a high off rate, might be a prerequisite for a successful positive selection of $\mathrm{T}$ cells in accordance with the affinity model of thymic selection. The affinity model states that low-affinity binding of the $\mathrm{T}$ cell receptor (TCR) to $\mathrm{MHC} /$ peptide complexes will lead to positive selection, whereas high-affinity TCR binding will lead to negative selection [56].

The structural, enzymatic and functional data on $\beta 5 \mathrm{t}$ all are remarkably consistent. There is only one experimental aspect that is difficult to reconcile with a large body of data. It has been shown that MHC class I ligands with weak Cterminal anchor residues are poor stabilizers of class I cell surface expression [57]. Nevertheless, a detailed analysis of surface expression of three different class I molecules on cTECs from wild type and $\beta 5 \mathrm{t}^{-/-}$mice revealed that the lack of $\beta 5 \mathrm{t}$ did not affect class I surface expression [55]. Perhaps cTECs have developed mechanisms that allow the stabilization even of weak peptide/class I/ $\beta 2$-microglobulin complexes on the cell surface. However, this and other hypotheses will be difficult to address experimentally because few cTECs can be isolated from a murine thymus.

\section{The spermatoproteasome in the generation of spermatids}

The most extensive tissue-specific alteration of proteasome subunit composition has been reported for the testes and in particular for spermatids [58]. The differentiation from spermatogonial stem cells to mature sperms is a highly regulated cellular process that includes mitosis, meiosis, and morphological changes, which are steps that might all be dependent on proteasomal function. The existence of testis-specific proteasome subunits was first described for Drosophila melanogaster in the mid-1990s. Belote and coworkers reported two alternative $\alpha 4$-type subunits $(\alpha 4 \mathrm{~T} 1$ and $\alpha 4 \mathrm{~T} 2$ ) encoded by paralogous genes that are exclusively expressed in the male germline [59]. Subsequent studies revealed that proteasome subunit gene duplications are widespread in $D$. melanogaster: to date, 12 of the $3326 \mathrm{~S}$ proteasome subunits have been identified to exist in two or even three different isoforms. Although the conventional form of each subunit can be detected in all tissues and during all developmental stages examined, all additional isoforms are specifically expressed in the testes (reviewed in [58]). Some alternative subunits were studied in more detail using reporter transgenes. These investigations showed an expression pattern limited to male germ line cells during the mid to late stages of spermatogenesis, whereas only the conventional proteasome subunits are expressed in the early gonial stages [59-61]. In elongated spermatids proteasomal 'speckles' containing alternative subunits could be observed in close proximity to the individualization complex (IC) [60] (Figure 3). The cytoskeletal membranous IC harbors a cluster of actin cones and mediates sperm individualization by moving down the spermatid bundle, thus expelling cytoplasm and organelles and simultaneously packing each spermatid in its own plasma membrane. The functional consequence of the proteasomal subcellular localization at the IC has not yet been clarified 

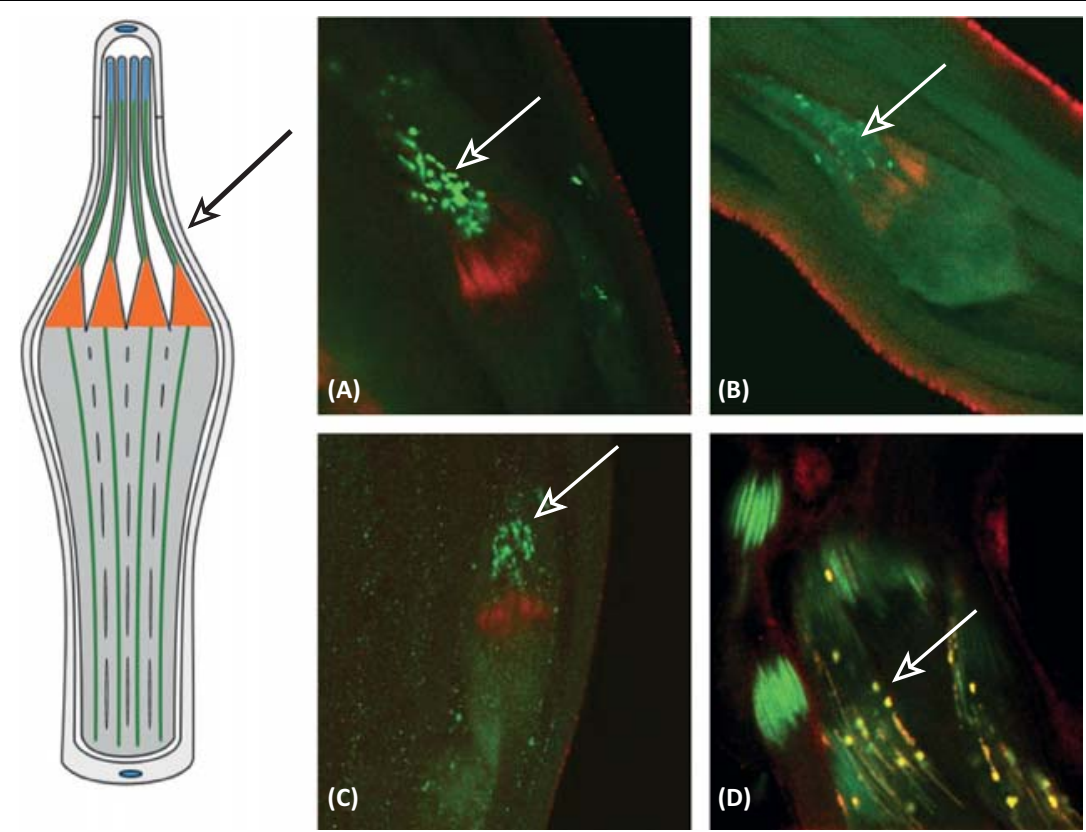

$\overline{\mathrm{T} i \mathrm{BS}}$

Figure 3. Images of testis-specific proteasome subunits $\alpha 3 T$ and $\alpha 6 \mathrm{~T}$ in the individualization complex (IC) of spermatids in Drosophila melanogaster. During the individualization of spermatids the syncytial spermatid bundle is resolved into separate sperm cells. On the left a cartoon of the IC is depicted; it is composed of actin cones (orange) with the cystic bulge, which is formed as the IC moves down the spermatid bundle. Sperm nuclei are at the top (blue). (A) Speckles of $\alpha 3$ T-GFP (green) trailing the actin cones (red). (B) Speckles of $\alpha 6$ T-GFP (green) trailing the actin cones (red). (C) Immunostaining of wild type testis with anti-proteasome antibodies (green). (D) Immunostaining of $\alpha 6 \mathrm{~T}$-GFP in the testis of an $\alpha 6 \mathrm{~T}$-GFP transgenic fly with anti-proteasome antibody showing the colocalization (yellow) of $\alpha 6 \mathrm{~T}$-GFP and proteasome signals. Reproduced, with permission, from [58].

but, interestingly, knockout of the alternative subunit $\alpha 6 \mathrm{~T}$ in D. melanogaster resulted in male sterility based on a defective sperm individualization, which was accompanied by disrupted actin cone coordination in ICs [60].

Recent work detected the alternative $\alpha 4$-type proteasome subunit, $\alpha 4 \mathrm{~s} / \mathrm{PSMA} 8$, in the mammalian testes. Its expression is restricted to spermatids and mature sperm, which correspond to the late stage expression of alternative subunits in D. melanogaster [62,63]. As the proteasomal outer $\alpha$ ring lacks catalytic activity, the function of alternative $\alpha$ subunits is less clear. One possibility could be a preferred interaction with certain regulatory particles. Besides $\alpha 4 \mathrm{~s}$, mammalian testis proteasomes were reported to contain immunoproteasome subunits $[63,64]$. However, $\beta 1 \mathrm{i}, \beta 2 \mathrm{i}$, and $\beta 5 \mathrm{i}$ showed partly dissimilar cell type and differentiation stage expression patterns. Clearly, lower amounts of immunoproteasomes could be detected in testes compared to spleen [63].

In addition to the special subunit composition of the $20 \mathrm{~S}$ complex, the associated regulatory particles are also testis specific. The proteasome regulator PA200 is expressed in different mammalian tissues but it is particularly abundant in testis [65], where PA200-capped proteasomes are present as single or double-capped complexes or in the form of hybrid proteasomes that carry both PA200 and the 19S regulator, each binding on opposing $\alpha$-endplates of the $20 \mathrm{~S}$ barrel [63]. The $200-\mathrm{kDa}$ PA200 is a monomeric non-ATPase proteasome activator that attaches to the outer $\alpha$ rings of the $20 \mathrm{~S}$ complex and enhances the proteasomal degradation of small peptides but not ubiquitylated protein substrates in vitro [65]. PA200 knockout mice are viable and show no obvious developmental abnormalities. However, PA200-deficient males exhibit a marked reduction of fertility due to defective spermatogenesis [66]. A mechanistic explanation was recently suggested when Qian et al. showed that PA200 promotes the acetylation-dependent degradation of core histones during somatic DNA damage responses and spermatogenesis [63]. Histone acetylation weakens the histoneDNA interaction and leads to an open and transcriptionally active chromatin structure. During spermatogenesis, histone acetylation is followed by removal of histones from chromatin and their replacement by transition proteins and subsequently protamines, which allow denser DNA packing [67]. An interesting question, which has not been addressed yet, is whether the testis-specific $\alpha 4$ s/PSMA8 subunit supports the docking of PA200 more than the conventional $\alpha 4$ subunit does. Taken together, PA200-dependent (and perhaps $\alpha 4$ s/PSMA 8 dependent) proteasomal degradation of acetylated histones might represent an important mechanism during spermatogenesis that enables transcription arrest, spermatid differentiation, and chromatin condensation.

\section{Concluding remarks and future perspectives}

The existence of tissue-specific proteasomes or proteasomes that are only expressed during stimulation with cytokines pose many unanswered questions. Are there selective substrates of tissue-specific proteasomes? Do tissue-selective proteasomes process precursor proteins in a different manner as compared to constitutive proteasomes? Do tissue-selective proteasomes associate differently with the increasing number of proteasome regulators? Apart from these fundamental biological questions tissue-specific proteasomes offer interesting new 


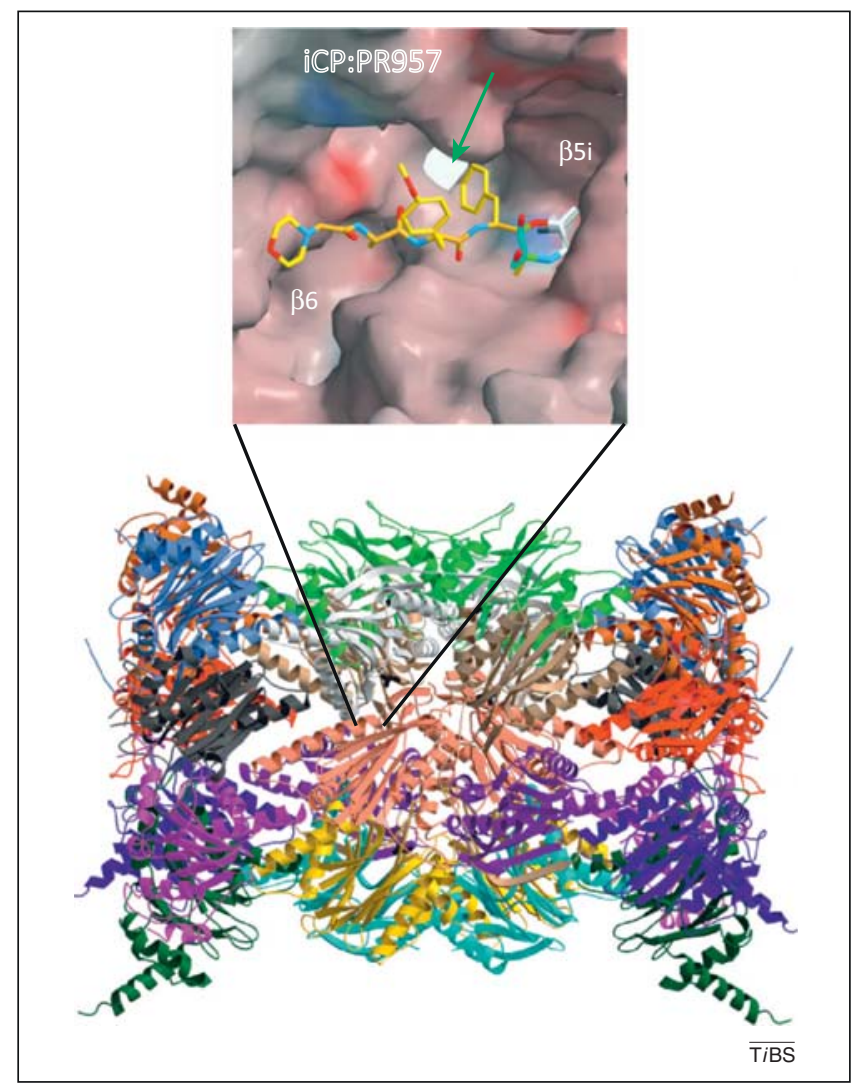

Figure 4. Structure of the mouse immunoproteasome with the $\beta 5 i$ (low molecular mass polypeptide 7; LMP7) active site bound by the $\beta 5$ i inhibitor ONX 0914 (formerly called PR-957). Surface colors in the Conolly surface representation of $\beta 5 \mathrm{i}$ indicate positive and negative electrostatic potentials contoured from $50 \mathrm{kT} / \mathrm{e}$ (intense blue) to $-50 \mathrm{kT} / \mathrm{e}$ (intense red). In order to illustrate conformational changes induced by the inhibitor, the amino acids $46-50$ were removed. Thr 1 of the $\beta 5 i$ subunit is colored in white and the covalently bound inhibitor in yellow. The green arrow indicates where the phenyl side chain of the inhibitor would clash with the constitutive $\beta 5$ subunit, whereas in the immunoproteasome subunit $\beta 5 \mathrm{i}$, a reorientation of the $\mathrm{CH} 3-\mathrm{S}$ - group of Met45 avoids such a steric clash, thus explaining the immunoproteasome selectivity of ONX 0914. Adapted, with permission, from $[13,72]$.

perspectives for their selective pharmacological inhibition while leaving the activity of constitutive proteasomes intact. Broad-spectrum proteasome inhibitors like bortezomib (Velcade) or carfilzomib (Kyprolis) are currently used to treat multiple myeloma but they need to be used close to the maximally tolerated dose to be effective. The dose-limiting side effects stem from the inhibition of the housekeeping functions of the constitutive proteasome needed in virtually all tissues [68]. Subunit-specific proteasome inhibitors, in contrast, may target diseases in a tissue specific manner. The $\beta 5 \mathrm{i}$ inhibitor ONX 0914 is a prominent example because it can suppress autoimmune diseases at doses far below the maximal tolerated dose in mice [37] (Figure 4). Other immunoproteasome-specific inhibitors such as UK-101 and IPSI, which both inhibit the $\beta 1 \mathrm{i}$ subunit, have been developed with the aim of improving cancer therapy $[69,70]$. Although cell-permeable inhibitors of the trypsin-like activity of the proteasome and immunoproteasome have been developed successfully, they do not discriminate between $\beta 2$ and $\beta 2$ i, probably because their substratebinding pocket is similar [71]. It will be interesting to follow the further development of subunit-selective inhibitors and to test their therapeutic potential in preclinical models and in humans. Apart from autoimmune diseases and cancer the in vivo manipulation of antigen presentation and $\mathrm{T}$ cell selection in the thymus might offer unanticipated clinical opportunities. Testis-specific proteasome subunits or regulators, by contrast, may serve as targets for the development of male contraceptives.

\section{Acknowledgments}

We thank John M. Belote for critical reading of the manuscript. This work was funded by the German Research Foundation grant GR1517/12-1, the Konstanz Research School Chemical Biology, the Fritz Thyssen Foundation grant AZ 10.10.2.122, and the Swiss National Science Foundation grant 31003A_138451.

\section{References}

1 Löwe, J. et al. (1995) Crystal structure of the $20 \mathrm{~S}$ proteasome from the archaeon T. acidophilum at 3.4 A resolution. Science 268, 533-539

2 Tamura, T. et al. (1995) The first characterization of a eubacterial proteasome: the 20S complex of Rhodococcus. Curr. Biol. 5, 766-774

3 Groll, M. et al. (1997) Structure of $20 \mathrm{~S}$ proteasome from yeast at 2.4A resolution. Nature 386, 463-471

4 Heinemeyer, W. et al. (1997) The active sites of the eukaryotic $20 \mathrm{~S}$ proteasome and their involvement in subunit precursor processing. $J$. Biol. Chem. 272, 25200-25209

5 Barton, L.F. et al. (2002) Regulation of immunoproteasome subunit expression in vivo following pathogenic fungal infection. J. Immunol. 169, 3046-3052

6 Kremer, M. et al. (2010) Reduced immunoproteasome formation and accumulation of immunoproteasomal precursors in the brains of lymphocytic choriomeningitis virus-infected mice. J. Immunol. 185, 5549-5560

7 Khan, S. et al. (2001) Immunoproteasomes largely replace constitutive proteasomes during an antiviral and antibacterial immune response in the liver. J. Immunol. 167, 6859-6868

8 Groettrup, M. et al. (2010) Proteasomes in immune cells: more than peptide producers? Nat. Rev. Immunol. 10, 72-77

9 Strehl, B. et al. (2005) Interferon-gamma, the functional plasticity of the ubiquitin- proteasome system, and MHC class I antigen processing. Immunol. Rev. 207, 19-30

10 Rock, K.L. et al. (1994) Inhibitors of the proteasome block the degradation of most cell proteins and the generation of peptides presented on MHC class I molecules. Cell 78, 761-771

11 Craiu, A. et al. (1997) Lactacystin and clasto-lactacystin beta-lactone modify multiple proteasome beta-subunits and inhibit intracellular protein degradation and major histocompatibility complex class I antigen presentation. J. Biol. Chem. 272, 13437-13445

12 Bai, A. and Forman, J. (1997) The effect of the proteasome inhibitor lactacystin on the presentation of transporters associated with antigen processing (TAP)-dependent and TAP-independent peptide epitopes by class I molecules. J. Immunol. 159, 2139-2146

13 Huber, E.M. et al. (2012) Immuno- and constitutive proteasome crystal structures reveal differences in substrate and inhibitor specificity. Cell $148,727-738$

14 Fehling, H.J. et al. (1994) MHC class I expression in mice lacking proteasome subunit LMP-7. Science 265, 1234-1237

15 Basler, M. et al. (2011) The antiviral immune response in mice devoid of immunoproteasome activity. J. Immunol. 187, 5548-5557

16 Kincaid, E.Z. et al. (2012) Mice completely lacking immunoproteasomes show major changes in antigen presentation. Nat. Immunol. 13, 129-135

17 Salzmann, U. et al. (1999) Mutational analysis of subunit i beta 2 (MECL-1) demonstrates conservation of cleavage specificity between yeast and mammalian proteasomes. FEBS Lett. 454, 11-15

18 Basler, M. et al. (2006) An altered T cell repertoire in MECL-1-deficient mice. J. Immunol. 176, 6665-6672

19 Moebius, J. et al. (2010) Immunoproteasomes are essential for survival and expansion of T cells in virus-infected mice. Eur. J. Immunol. 40 3439-3449

20 Basler, M. et al. (2010) Prevention of experimental colitis by a selective inhibitor of the immunoproteasome. J. Immunol. 185, 634-641

21 Sijts, A.J.A.M. et al. (2000) MHC class I antigen processing of an Adenovirus CTL epitope is linked to the levels of immunoproteasomes in infected cells. J. Immunol. 164, 4500-4506 
22 Palmowski, M.J. et al. (2006) Role of immunoproteasomes in crosspresentation. J. Immunol. 177, 983-990

23 Chen, W.S. et al. (2001) Immunoproteasomes shape immunodominance hierarchies of antiviral CD8 ${ }^{+} \mathrm{T}$ cells at the levels of $\mathrm{T}$ cell repertoire and presentation of viral antigens. J. Exp. Med. 193, 1319-1326

24 Hutchinson, S. et al. (2011) A dominant role for the immunoproteasome in $\mathrm{CD} 8+\mathrm{T}$ cell responses to murine cytomegalovirus. PLoS ONE 6, e14646

25 Basler, M. et al. (2004) Immunoproteasomes down-regulate presentation of a subdominant $\mathrm{T}$ cell epitope from lymphocytic choriomeningitis virus. J. Immunol. 173, 3925-3934

26 Morel, S. et al. (2000) Processing of some antigens by the standard proteasome but not by the immunoproteasome results in poor presentation by dendritic cells. Immunity 12, 107-117

27 Basler, M. et al. (2012) Why the structure but not the activity of the immunoproteasome subunit LMP2 rescues antigen presentation. $J$. Immunol. 189, 1868-1877

28 deVerteuil, D. et al. (2010) Deletion of immunoproteasome subunits imprints on the transcriptome and has a broad impact on peptides presented by major histocompatibility complex I molecules. Mol. Cell. Proteomics 9, 2034-2047

29 Strehl, B. et al. (2006) Immunoproteasomes are essential for clearance of Listeria monocytogenes in nonlymphoid tissues but not for induction of bacteria-specific CD8 ${ }^{+}$T cells. J. Immunol. 177, 6238-6244

$30 \mathrm{Tu}$, L. et al. (2009) Critical role for the immunoproteasome subunit LMP7 in the resistance of mice to Toxoplasma gondii infection. Eur. J. Immunol. 39, 3385-3394

31 Groettrup, M. et al. (2001) Interferon- $\gamma$ inducible exchanges of $20 \mathrm{~S}$ proteasome active site subunits: Why? Biochimie 83, 367-372

32 Zaiss, D.M. et al. (2011) Proteasome immunosubunits protect against the development of CD8 T cell-mediated autoimmune diseases. J. Immunol. 187, 2302-2309

33 Pryhuber, K.G. et al. (1996) Polymorphism in the LMP2 gene influences disease susceptibility and severity in HLA-B27 associated juvenile rheumatoid arthritis. J. Rheumatol. 23, 747-752

34 Kitamura, A. et al. (2011) A mutation in the immunoproteasome subunit PSMB8 causes autoinflammation and lipodystrophy in humans. J. Clin. Invest. 121, 4150-4160

35 Arima, K. et al. (2011) Proteasome assembly defect due to a proteasome subunit beta type 8 (PSMB8) mutation causes the autoinflammatory disorder, Nakajo-Nishimura syndrome. Proc. Natl. Acad. Sci. U.S.A. 108, 14914-14919

36 Agarwal, A.K. et al. (2010) PSMB8 encoding the beta 5i proteasome subunit is mutated in joint contractures, muscle atrophy, microcytic anemia, and panniculitis- induced lipodystrophy syndrome. Am. $J$ Hum. Genet. 87, 866-872

37 Muchamuel, T. et al. (2009) A selective inhibitor of the immunoproteasome subunit LMP7 blocks cytokine production and attenuates progression of experimental arthritis. Nat. Med. 15, 781-787

38 Schmidt, N.et al. (2010) Targeting the proteasome: partial inhibition of the proteasome by bortezomib or deletion of the immunosubunit LMP7 attenuates experimental colitis. Gut 59, 896-906

39 Fitzpatrick, L.R. et al. (2006) Dextran sulfate sodium-induced colitis is associated with enhanced low molecular mass polypeptide 2 (LMP2) expression and is attenuated in LMP2 knockout mice. Digest Dis. Sci. 51, 1269-1276

40 Ichikawa, H.T. et al. (2012) Beneficial effect of novel proteasome inhibitors in murine lupus via dual inhibition of type I interferon and autoantibody-secreting cells. Arthritis. Rheum. 64, 493503

41 Kalim, K.W. et al. (2012) Immunoproteasome subunit LMP7 deficiency and inhibition suppresses Th1 and Th17 but enhances regulatory T cell differentiation. J. Immunol. 189, 4182-4193

42 Reis, J. et al. (2011) The immunoproteasomes regulate LPS-induced TRIF/TRAM signaling pathway in murine macrophages. Cell Biochem. Biophys. 60, 119-126

43 Palombella, V.J. et al. (1994) The ubiquitin-proteasome pathway is required for processing the NF-кB1 percursor protein and the activation of NF-кB. Cell 78, 773-785

44 Hoppe, T. et al. (2000) Activation of a membrane-bound transcription factor by regulated ubiquitin/proteasome-dependent processing. Cell $102,577-586$
45 Hayashi, T. and Faustman, D. (1999) NOD mice are defective in proteasome production and activation of NF-kappa B. Mol. Cell. Biol. 19, 8646-8659

46 Kessler, B.M. et al. (2000) LMP2 expression and proteasome activity in NOD mice. Nat. Med. 6, 1064

47 Runnels, H.A. et al. (2000) LMP2 expression and proteasome activity in NOD mice. Nat. Med. 6, 1064-1065

48 Seifert, U. et al. (2010) Immunoproteasomes preserve protein homeostasis upon interferon-induced oxidative stress. Cell 142, $613-624$

49 Nathan, J.A. et al. (2013) Immuno- and constitutive proteasomes do not differ in their abilities to degrade ubiquitinated proteins. Cell 152, 1184-1194

50 Prakash, S. et al. (2004) An unstructured initiation site is required for efficient proteasome- mediated degradation. Nat. Struct. Mol. Biol. 11, 830-837

51 Murata, S. et al. (2007) Regulation of $\mathrm{CD}^{+} \mathrm{T}$ cell development by thymus-specific proteasomes. Science 316, 1349-1353

52 Klein, L. et al. (2009) Antigen presentation in the thymus for positive selection and central tolerance induction. Nat. Rev. Immunol. 9, 833-844

$53 \mathrm{Nil}, \mathrm{A}$. et al. (2004) Expression of housekeeping and immunoproteasome subunit genes is differentially regulated in positively and negatively selecting thymic stroma subsets. Eur. J. Immunol. 34, 2681-2689

54 Macagno, A. et al. (1999) Dendritic cells upregulate immunoproteasomes and the proteasome regulator PA28 during maturation. Eur. J. Immunol. 29, 4037-4042

55 Nitta, T. et al. (2010) Thyrnoproteasorne shapes immunocompetent repertoire of $\mathrm{CD}^{+} \mathrm{T}$ cells. Immunity $32,29-40$

56 Murata, S. et al. (2008) Thymoproteasome: probable role in generating positively selecting peptides. Curr. Opin. Immunol. 20, 192-196

57 Rammensee, H.G. et al. (1993) Peptides naturally presented by MHC class I molecules. Annu. Rev. Immunol. 11, 213-244

58 Belote, J.M. and Zhong, L. (2009) Duplicated proteasome subunit genes in Drosophila and their roles in spermatogenesis. Heredity 103, 23-31

59 Yuan, X. et al. (1996) Duplicated proteasome subunit genes in Drosophila melanogaster encoding testes-specific isoforms. Genetics 144, 147-157

60 Zhong, L. and Belote, J.M. (2007) The testis-specific proteasome subunit Pros alpha 6T of D-melanogaster is required for individualization and nuclear maturation during spermatogenesis. Development 134, 3517-3525

$61 \mathrm{Ma}$, J.et al. (2002) Expression of proteasome subunit isoforms during spermatogenesis in Drosophila melanogaster. Insect Mol. Biol. 11, 627-639

62 Skerget, S. et al. (2013) The rhesus macaque (Macaca mulatta) sperm proteome. Mol. Cell. Proteomics 1, 1

63 Qian, M.X. et al. (2013) Acetylation-mediated proteasomal degradation of core histones during DNA repair and spermatogenesis. Cell 153, 1012-1024

64 Sutovsky, P. et al. (2004) Proteasomal interference prevents zona pellucida penetration and fertilization in mammals. Biol. Reprod. $71,1625-1637$

65 Ustrell, V.et al. (2002) PA200, a nuclear proteasome activator involved in DNA repair. EMBO J. 21, 3516-3525

66 Khor, B. et al. (2006) Proteasome activator PA200 is required for normal spermatogenesis. Mol. Cell. Biol. 26, 2999-3007

67 Gaucher, J.et al. (2010) From meiosis to postmeiotic events: the secrets of histone disappearance. FEBS J. 277, 599-604

68 Huber, E.M. and Groll, M. (2012) Inhibitors for the immuno- and constitutive proteasome: current and future trends in drug development. Angew. Chem. Int. Ed. Engl. 51, 8708-8720

69 Ho, Y.K. et al. (2007) LMP2-specific inhibitors: chemical genetic tools for proteasome biology. Chem. Biol. 14, 419-430

70 Kuhn, D.J. et al. (2009) Targeted inhibition of the immunoproteasome is a potent strategy against models of multiple myeloma that overcomes resistance to conventional drugs and nonspecific proteasome inhibitors. Blood 113, 4667-4676

71 Mirabella, A.C. et al. (2011) Specific cell-permeable inhibitor of proteasome trypsin-like sites selectively sensitizes myeloma cells to bortezomib and carfilzomib. Chem. Biol. 18, 608-618

72 Basler, M. et al. (2013) The immunoproteasome in antigen processing and other immunological functions. Cur. Opin. Immunol. 25, 74-80 